\title{
Painleve Analysis and Symmetries of the Hirota-Satsuma Equation
}

\author{
A.A. MOHAMMAD $D^{\dagger}$ and M. CAN \\ Istanbul Technical University, Mathematics Department,

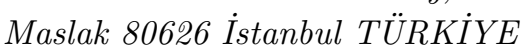

\begin{abstract}
The singular manifold expansion of Weiss, Tabor and Carnevale [1] has been successfully applied to integrable ordinary and partial differential equations. They yield information such as Lax pairs, Bäcklund transformations, symmetries, recursion operators, pole dynamics, and special solutions. On the other hand, several recent developments have made the application of group theory to the solution of the differential equations more powerful then ever. More recently, Gibbon et. al. [2] revealed interrelations between the Painlevè property and Hirota's bilinear method. And W. Strampp [3] hase shown that symmetries and recursion operators for an integrable nonlinear partial differential equation can be obtained from the Painlevè expansion. In this paper, it has been shown that the Hirota-Satsuma equation passes the Painlevé test given by Weiss et al. for nonlinear partial differential equations. Furthermore, the data obtained by the truncation technique is used to obtain the symmetries, recursion operators, some analytical solutions of the Hirota-Satsuma equation.
\end{abstract}

\section{Introduction}

The Hirota-Satsuma (H-S) equation

$$
\begin{aligned}
& u_{t}=u_{x x x} / 2+3 u u_{x}-3 v v_{x}, \\
& v_{t}=-v_{x x x}-3 u v_{x}
\end{aligned}
$$

is classified as a soliton equation by B. Fuchssteiner [4]. B. Fuchssteiner also claims that this equation has a bi-hamiltonian formulation and obtains countably many conserved quantities and symmetry generators. Complete integrability of this equation is conjectured by Hirota and Satsuma.

In this paper, we will see that the Painlevé analysis is a powerful tool for construction of symmetries, explicit solutions and Lie-Bäcklund transformations. It also helps to find Lax pairs and recursion operators and plays an important role in the study of a chaotic behaviour of nonlinear partial differential equations.

Copyright (c) 1996 by Mathematical Ukraina Publisher. All rights of reproduction in any form reserved.

\footnotetext{
${ }^{\dagger}$ Permanent addres: Garyunis University, Mathematics Department, Bengazi, LIBYA.
} 


\section{Painlevé analysis for the $\mathrm{H}-\mathrm{S}$ equation}

Let $\phi(x, t)=0$ be the solution singularity manifold of (1) and

$$
\begin{aligned}
& u=\phi^{p} \sum_{j=0}^{\infty} u_{j} \phi^{j}, \\
& v=\phi^{p} \sum_{j=0}^{\infty} v_{j} \phi^{j} .
\end{aligned}
$$

Substituting (2) into (1), we have $p=-2$, resonances at $j=-1,1,2,4$ and a double resonance at $j=6$. Hence if the compatibility conditions hold, we can have six arbitrary functions to write down a general solution of (1). From the recurrence relations, we also have

$$
\begin{aligned}
& u_{0}=-2 \phi_{x}^{2}, \quad v_{0}=0 \\
& u_{1}=2 \phi_{x x}, \quad v_{1} \text { arbitrary; } \\
& u_{2}=\left(-3 v_{1}^{2}+6 \phi x x^{2}-8 \phi_{x} \phi_{x x x}+4 \phi x \phi_{t}\right) / 12 \phi_{x}^{2}, \quad v_{2} \text { arbitrary. }
\end{aligned}
$$

The coefficient functions $u_{4}, u_{6}$ and $v_{6}$ are found to be arbitrary and the corresponding compatibility conditions are satisfied identically for the above values of $u_{0}, v_{0}, u_{1}$ and $u_{2}$. Hence, the system of quasilinear partial differential equations (1) passes the Painlevé test for PDE's.

In the following we are going to use the above results to derive some valuable facts for the $\mathrm{H}-\mathrm{S}$ equation.

\section{Exact solutions of the $\mathrm{H}-\mathrm{S}$ equation}

Let us truncate the series in (2) at the second term and assume that $u_{j}=0, j \geq 3$. Then we have

$$
\begin{aligned}
& u=\frac{u_{0}}{\phi^{2}}+\frac{u_{1}}{\phi}+u_{2}, \\
& v=\frac{v_{0}}{\phi}+\frac{v_{1}}{\phi}+v_{2} .
\end{aligned}
$$

If we let $u_{3}=0$ in the recurrence relation, the right-hand side also vanishes and this assumption brings no restriction on the unknowns. The assumption $v_{3}=0$ leads to

$$
3 \phi_{x} v_{1 x x}-3 \phi_{x x} v_{1 x}-\left(3 \phi_{x} u_{2}-\phi_{x x x}+\phi_{t}\right) v_{1}+6 \phi_{x}^{2} v_{2 x}=0 .
$$

Meanwhile $u_{3}=v_{3}=u_{4}=v_{4}=0$ yields

$$
-3\left(v_{1} v_{2}\right)_{x}+3\left(u_{1} u_{2}\right)_{x}+\frac{1}{2} u_{1 x x x}-u_{1 t}=0,
$$

and

$$
v_{1 x x x}+3 u_{2} v_{1 x}+v_{1 t}+3 v_{2 x} u_{1}=0 .
$$

On the other hand, $u_{3}=v_{3}=u_{4}=v_{4}=u_{5}=v_{5}=0$ yields the system

$$
\begin{aligned}
& u_{2 t}=u_{2 x x x} / 2+3 u_{2} u_{2 x}-3 v_{2} v_{2 x}, \\
& v_{2 t}=-v_{2 x x x}-3 u_{2} v_{2 x} .
\end{aligned}
$$


Hence, $u_{2}, v_{2}$ must be a solution of the H-S equation (1). Hence (4) is an auto Bäcklund transformation of the $\mathrm{H}-\mathrm{S}$ equation. To find a truncated exact solution of the $\mathrm{H}-\mathrm{S}$ equation, one needs to solve the equations (5), (6), (7) and (8) for $\phi, v_{1}$ and $v_{2}$. This is an over-determined system and in what follows we are going to find some special solutions.

\section{Some special solutions}

Let $u_{2}(t, x)=A$ and $v_{2}(t, x)=B$ be the constant solution of system (8) and let $v_{1}(t, x)=0$. Then equations (5) and (7) are satisfied automatically and (6) leads to

$$
6 A \phi_{x x}+\phi_{x x x x}-2 \phi_{x t}=0,
$$

and the formula for $u_{2}(t, x)$ reduces to

$$
12 A \phi_{x}^{2}=6 \phi x x^{2}-8 \phi_{x} \phi_{x x x}+4 \phi x \phi_{t} .
$$

We can integrate (9) once in $x$ to get

$$
\phi_{x x x}+6 A \phi_{x}-2 \phi_{t}=-2 C^{\prime}(t), \quad '=\frac{d}{d t} .
$$

The equation (10) is a partial differential equation with constant coefficients and has a general solution of the form

$$
\phi(x, t)=C(t)+e^{k\left(x+\left(k^{2}+6 A\right) / 2 t\right)}
$$

where $k$ is a complex number and $C(t)$ is an arbitrary function. To determine them, let us substitute this solution into (10). This gives $C^{\prime}(t)=0$ and hence $C(t)=C$ is a constant. Using this result for $\phi(t, x)$, one can find the truncated solution (4) from the formula

$$
\begin{aligned}
& u=\frac{-2 \phi_{x}^{2}}{\phi^{2}}+\frac{2 \phi_{x x}}{\phi}+A, \\
& v=B .
\end{aligned}
$$

Substituting this set of two functions into the $\mathrm{H}-\mathrm{S}$ equation (1), we find a dispersion relation between $k$ and $A: k^{2}-24 A=0$. Hence a special solution for the $\mathrm{H}-\mathrm{S}$ equation is given by

$$
\begin{aligned}
u & =2 k^{2} C \frac{e^{k \zeta}}{\left(C+e^{k \zeta}\right)^{2}}+A, \quad \zeta=x+5 k^{2} t / 8 . \\
v & =B,
\end{aligned}
$$

\section{$5 \quad$ Symmetry by truncated expansions}

Let us write system (1) as

$$
\begin{aligned}
& u_{t}=F(u, v)=u_{x x x} / 2+3 u u_{x}-3 v v_{x} \\
& v_{t}=G(u, v)=-v_{x x x}-3 u v_{x} .
\end{aligned}
$$

To find the symmetries, we need the linearization of (15) about a solution $u, v$;

$$
\begin{aligned}
& w_{1 t}=\left.\frac{\partial}{\partial \varepsilon} F\left(u+\varepsilon w_{1}, v+\varepsilon w_{2}\right)\right|_{\varepsilon=0}=w_{1 x x x} / 2+3\left(u w_{1}\right)_{x}-3\left(v w_{2}\right)_{x}, \\
& w_{2 t}=\left.\frac{\partial}{\partial \varepsilon} G\left(u+\varepsilon w_{1}, v+\varepsilon w_{2}\right)\right|_{\varepsilon=0}=-w_{2 x x x}-3 u w_{2 x}-3 w_{1} v_{x} .
\end{aligned}
$$


Any solution $w_{1}, w_{2}$ of (16) yields a symmetry or infinitesimal transformation about $u, v$. That is, the transformation

$$
\begin{aligned}
t^{*} & =t, \\
x^{*} & =x, \\
u^{*} & =u+\varepsilon w_{1}, \\
v^{*} & =v+\varepsilon w_{2}
\end{aligned}
$$

leaves the system (1) form-invariant.

Comparing (16) with (6) and (7), we see that $u_{1}, v_{1}$ is the solution of the linearized system about $u_{2}, v_{2}$. Hence, any solution of the system formed by equations (5), (6), (7) and (8) for $\phi, v_{1}$ and $v_{2}$ gives a symmetry of the $\mathrm{H}-\mathrm{S}$ equation.

\section{Discussion}

The Painlevé analysis provides a new and powerful tool for constructing explicit solutions for nonintegrable as well as integrable dynamical systems. But it only gives possible solutions, so one must check the results if they are actual solutions of the given nonlinear partial differential equation. On the other hand, in general the necessary calculations are too tedious to do by hand. In those cases, we interacted with some packages just like MATHEMATICA.

\section{References}

[1] Weiss J., Tabor M. and Carnevale G., J. Math. Phys., 1983, V.24, 522.

[2] Gibbon J.D., Radmore P., Tabor M. and Wood D., Stud. Appl. Math., 1985, V.72, 39.

[3] Strampp W., Prog. of Theoretical Phys., 1986, V.76, N 4, 802.

[4] Fuchssteiner B., Prog. of Theoretical Phys., 1982, V.68, N 4, 1082. 